\title{
Evaluación de la vivienda de interés prioritario desde la perspectiva de los beneficiarios: urbanización Antonia Santos, Tunja, Colombia
}

Juan M. Mendoza-Vargas' ${ }^{1}$, Víctor M. Burbano-Pantoja ${ }^{\star}$, y Hugo H. Mendoza-Vargas ${ }^{3}$

(1) Univ. Pedagógica y Tecnológica de Colombia, Uptc. Av Central del Norte, Tunja Colombia. (correo-e: juanmaria.mendoza@uptc.edu.co)

(2) Facultad de Ciencias, Escuela de matemáticas, Univ. Pedagógica y Tecnológica de Colombia, Uptc. Av Central del Norte, Tunja Colombia. (correo-e: victor.burbano@uptc.edu.co)

(3) Univ. Pedagógica y Tecnológica de Colombia, Uptc. Av Central del Norte, Tunja Colombia. (correo-e:2hsmendoza@gmail.com)

* Autor a quien debe ser dirigida la correspondencia.

Recibido Feb. 4, 2021; Aceptado Abr. 5, 2021; Versión final May. 6, 2021, Publicado Ago. 2021

\section{Resumen}

El objetivo del presente trabajo investigativo se centró en evaluar la vivienda de interés prioritario (VIP) desde la perspectiva de un grupo de beneficiarios del programa de vivienda gratuita, proyecto Antonia Santos, implementado por el municipio de Tunja, Colombia. La metodología asume un enfoque cuantitativo de investigación apoyado con exégesis textual; los datos fueron recolectados mediante encuesta a 72 beneficiarios. El procesamiento de la información se efectuó usando el software estadístico R. Los resultados mostraron que los usuarios valoran en un nivel regular el estado de la VIP respecto a sus características físicas, ambientales, funcionales y de sustentabilidad. Se concluye que la VIP mitiga la necesidad de techo para las familias vulnerables beneficiadas pero requiere mejorarse en diversos aspectos antes de adjudicarse.

Palabras clave: perspectiva; vivienda de interés prioritario; estado; VIP; calidad

\section{Evaluation of priority interest housing from the perspective of the beneficiaries: Antonia Santos urbanization, Tunja, Colombia}

\begin{abstract}
The objective of this research study was to evaluate priority interest housing (VIP, in Spanish) from the perspective of a group of beneficiaries of a free housing program, the Antonia Santos urbanization, implemented by the municipality of Tunja, Colombia. The methodology applied a quantitative research approach supported with textual exegesis. The data were collected through a survey of 72 beneficiaries. Data were processed by using the statistical software $R$. The results showed that users value at an average level their VIP status with respect to their VIP's physical, environmental, functional, and sustainability characteristics. It is concluded that VIP mitigated the need for housing of vulnerable families, but it requires improvement in various aspects before being offered.
\end{abstract}




\section{INTRODUCCIÓN}

La Constitución Nacional colombiana de 1991, en su artículo 51 pone de manifiesto el derecho que tienen las personas de poseer una vivienda digna; en este sentido, los gobiernos de turno hacen esfuerzos fiscales para hacer efectivo este mandato constitucional (Mendoza et al., 2020), tal derecho social y económico ha de estar sujeto al principio de progresividad y no regresividad; además, el estado a través de sus entes territoriales diseña y desarrolla programas que también garantizan soluciones de vivienda destinadas a los habitantes con baja capacidad económica, asimismo, el estado ha implementado políticas públicas para que paulatinamente más familias puedan acceder a su vivienda propia, crecer y desarrollarse tanto en lo económico como en lo social. Además, los programas de VIP han de beneficiar a los sujetos de especial protección constitucional, las personas desplazadas por violencia, los padres y madres cabeza de familia, adultos mayores, personas que vivan en zonas de alto riesgo, los damnificados, las familias en extrema pobreza, y poblaciones vulnerables, entre otros (Mejía, 2012; Serrano et al., 2019).

La política de VIP incluye programas que nacen bajo el mecanismo de la articulación entre el estado y los entes territoriales, los cuales son administrados bajo los lineamientos del artículo 209 de la constitución colombiana de 1991; posteriormente, la Ley 1537 del año 2012 estableció que la VIP podía ser otorgada a personas con especial protección constitucional a manera de subsidio en especie, sin tener que hacer pagos adicionales asociados con el precio del inmueble otorgado, sin necesidad del requisito de tener ahorro programado y sin asumir deuda con las entidades financieras encargadas del programa VIP. En este contexto, el municipio de Tunja mediante convocatoria No. 038 de octubre de 2012 da inicio al programa de vivienda gratuita con el proyecto de vivienda Antonia Santos, ubicado en la periferia de Tunja en la carrera 8 números 1 A-60 y 2-14, para construir inicialmente 452 soluciones VIP de un total de 792, bajo la tipología de vivienda multifamiliar con un área de 52.71 metros cuadrados, bajo la modalidad de construcción a precio global fijo, sin exceder los 70 salarios mínimos legales vigentes (SMLV). Así entonces, las características físicoespaciales, funcionales y ambientales de la VIP fueron proporcionales con su costo, en este marco, la pregunta que orientó el proceso investigativo fue ¿Cómo los beneficiarios evalúan la VIP que les fue adjudicada mediante el proyecto Antonia Santos en la ciudad de Tunja en Colombia?

La respuesta a este interrogante se fundamenta en un marco contextual que permite dilucidar algunos aspectos valorados por los usuarios en torno a la VIP que actualmente habitan, el contexto del barrio y las realidades socioeconómicas (condiciones actuales) por las cuales atraviesan las familias beneficiarias. De acuerdo con la Ley 1450 de 2011, uno de los parámetros para las constructoras que desarrollan proyectos VIP en Colombia, es que el valor de cada VIP no ha de superar los 70 SMLV (Chávez et al., 2018); este aspecto limita las condiciones físico-espaciales de las VIP y el proyecto Antonia Santos no ha sido la excepción, para este proyecto, ese valor también cubre las obras de urbanismo y construcción de la VIP, diseños arquitectónicos y estructurales, redes hidráulicas y sanitarias internas, conexiones eléctricas, redes de gas domiciliario y puntos de telecomunicaciones como se indica en la Convocatoria No. 038.

Respecto a la calidad de la VIP Murray y Clapham (2015) afirman que la calidad no solamente se debe limitar a los ambientes físicos construidos en cuanto a los espacios, sino también al componente social refiriéndose al grado de satisfacción que tienen los usuarios sobre el espacio en el que se habita; por su parte, Ceballos y Caquimbo (2014) indican que, otro aspecto a tener en cuenta es la calidad y el amueblamiento de cada espacio que conforma el hábitat familiar, los amoblados y el entorno de la vivienda generan satisfacción por el espacio y el uso correcto donde se vive, constituyéndose en un elemento que favorece el clima organizacional de la urbanización y el desempeño de quienes viven allí (Brito et al., 2020). En este escenario las dimensiones de la VIP reflejan las relaciones existentes entre las actividades diarias como son las de comer, cocinar, dormir y asearse, entre otras, y los hábitos del ser humano; tales hábitos se asocian también con la movilidad, la infraestructura del transporte, los sitios de trabajo, los espacios públicos y arquitectónicos, por esto, el usuario espera vivir en una VIP de calidad.

Aunque la VIP colombiana sea de bajo costo, los beneficiarios esperan se les entregue unas viviendas de buena calidad que se ajusten a sus necesidades y que en el corto plazo, no se observen como ruinas en una urbanización que desentone con la arquitectura de la ciudad, que mejore su calidad de vida y no genere insatisfacción familiar (Mejía, 2012). Otro aspecto a tener en cuenta y que afecta directamente al usuario es el promedio de integrantes por familia, en varios casos, la VIP ha de ser habitada por grupos familiares numerosos, con presencia de hacinamiento; por otro lado, los ingresos de las familias resultan insuficientes para hacerle mejoras a su VIP. Con frecuencia, las VIP tienen las mismas condiciones internas espaciales y son adjudicados por sorteo sin tener en cuenta las particularidades de las familias. El beneficiario expresa su satisfacción por la VIP cuando ésta presenta un buen aspecto, es espaciosa, el entorno físico y sus vecinos son agradables y se puede conectar con facilidad con el resto de la ciudad (Greif, 2015).

Von-Breymann (2019) indica que, algunos usuarios de VIP tienen una valoración particular sobre la gestión urbana y territorial efectuada por el gobierno local y muestran una satisfacción parcial respecto a variables 
como infraestructura, seguridad, solidaridad, uso del espacio, situación residencial y percepciones sobre sus vecinos y el barrio; al respecto, se ha diagnosticado que, los servicios básicos, el área socioeconómica y la infraestructura eran factores que influían directamente en la insatisfacción de ciertas personas sobre el área donde vivían. En este sentido Adewale et al., (2020) mencionan que también la insatisfacción de los usuarios está en el entorno del vecindario y las vías precarias de acceso a las urbanizaciones, los escasos servicios públicos y los factores asociados con la seguridad de sus habitantes. Por otra parte, Grasser et al., (2016) encontraron una asociación negativa entre la transitividad y el vecindario, la primera entendida como el espacio usado para el desarrollo de una serie de actividades, entre ellas, las recreativas, el acceso a diversos servicios y el tránsito hacia el lugar de trabajo; mientras las familias de estrato alto gozan de movilidad propia, quienes habitan barrios pobres, lo hacen en transporte público (Cardona eta al., 2020).

Por otra parte, en ciudades de América Latina, la construcción de vivienda social para familias con bajo ingreso sin excluir la VIP, ha sido bastante cuestionada tanto en la calidad del producto como también por su ubicación periférica (Rodríguez, 2020). De acuerdo con Libertun (2018), la periferia genera segregación e insatisfacción residencial, puede debilitar los vínculos sociales e incrementar los tiempos para que las personas se trasladen desde su residencia a los lugares trabajo; sin embargo, la construcción de VIP en áreas periféricas se justifica por un menor costo del suelo y la preferencia de los constructores por la economía de escala (Caldeira, 2017). Otros aspectos como la accesibilidad a la VIP, las dinámicas económicas de cada ciudad y la planificación urbana han confinado a la VIP a zonas periféricas; como era de esperarse Tunja no fue la excepción, el proyecto Antonia Santo se construyó en una zona periférica a la ciudad, los beneficiarios accedieron a su VIP por sorteo después de postularse y superar los requerimientos de una convocatoria pública realizada por el municipio para personas de especial protección constitucional.

\section{OTROS ANTECEDENTES}

Los planificadores urbanos en Tunja han hecho uso de La ley 388 para atender las dinámicas de crecimiento de la ciudad, sin desconocer el crecimiento histórico del entorno periférico de esta ciudad intermedia de Colombia, ni los derechos de los beneficiarios VIP (Hidalgo, 2008), esta ley es una normativa que desarrolla el ordenamiento del territorio municipal desde una perspectiva técnica, que engloba un conjunto de materias, entre ellas, el uso del suelo para hacer efectivos los derechos constitucionales a la vivienda de acuerdo con la Ley 9 de 1989, la defensa del espacio público, la prevención de desastres, la planificación urbanística eficiente y la realización de actuaciones urbanas integrales en consonancia con la política urbana nacional tendiente a mejorar la calidad de vida de sus ciudadanos. En este contexto, el proyecto Antonia Santos brindó la posibilidad de que un buen número de personas con especial protección constitucional pudieran acceder a una VIP. Los beneficiarios VIP fueron personas de la ciudad o que migraron a ésta por diversos motivos: el desplazamiento forzado, vivir en sitios de alto riesgo, familias damnificadas, vulnerables o en pobreza extrema y los cabeza de familia sin vivienda propia, entre otros.

El desplazamiento forzado se ha generado por la presión ejercida por grupos al margen de la ley; para mitigar algunos de sus efectos, el gobierno colombiano y los municipios han asignado algunos cupos de VIP 0 alternativas de ingresos económicos para que los desplazados accedan a una vivienda (Camargo et al., 2020). Esta situación se complejizó con la migración de familias campesinos en alto riesgo por desastres naturales o pobreza extrema, quienes buscaron mejores oportunidades en la ciudad, confiados en su capacidad de resiliencia o en un posible amparo del gobierno municipal (Malerba, 2020) pero acrecentando la construcción periférica o la falta de vivienda (Hidalgo, 2008); en este sentido, el municipio ha contribuido a menguar el déficit cuantitativo de vivienda de acuerdo con el principio de progresividad; con frecuencia, estas eventualidades exceden el crecimiento urbanístico proyectado en los planes de desarrollo territorial (Castles, 2014) y crean descontento en quienes no han sido beneficiados en la adjudicación de VIP.

Además, las personas cabeza de hogar también han de tenerse en cuenta en los procesos adjudicatorios de VIP; se trata de ciudadanos que como otros protegidos por la ley, con cierta resiliencia han afrontado sus necesidades prioritarias, los embates del desplazamiento o el riesgo por fenómenos naturales, entre otros (De la Ossa et al., 2018); en estas circunstancias, Naef (2020) sostiene que, la resiliencia entendida como la capacidad de las personas para sobreponerse a las dificultades, genera vínculos de cooperación familiar y social tendientes a mitigar sus principales necesidades, adquirir una vivienda propia, luchar contra la vulnerabilidad y la segregación tanto espacial como social o salir de la pobreza (Zhang y Li, 2018); como beneficiarios de los programas VIP esperan que su vivienda haya sido construida con buenos materiales y altos estándares de calidad (Castaño et al., 2018) y dentro de una política responsable de la sostenibilidad y sustentabilidad urbana (Wachsmuth y Angelo, 2018); sin embargo, la calidad de la vivienda en Colombia sea VIP u otra no es óptima porque los constructores privados piensan en el lucro con mínimo costo y llevan al límite la capacidad de carga de los materiales, generando riesgos para los usuarios (Ramírez, 2020).

En la VIP adjudicada, la interacción entre diversos grupos sociales genera complejos tipos de tensión social porque se conjugan diferentes costumbres e ideologías, distintas formas de sobreponerse a sus dificultades 
y de afrontar las necesidades básicas de su vida diaria; asimismo, emergen variadas preocupaciones focalizadas tanto en su bienestar físico como psicológico asociadas con el hecho de vivir en zonas periféricas o haber sido víctimas del conflicto armado (Castro et al., 2019), de poseer o no un empleo, de contar con vías y medios de transporte adecuados para conectarse con la ciudad o simplemente disfrutar de un ambiente residencial seguro, sustentable, agradable, sostenible y acorde a sus necesidades prioritarias. Esto implica que el municipio y la nación además de proveer la VIP para estos ciudadanos, también han de apoyar su bienestar psicológico y social generando espacios de mejora cualitativa de la vivienda, de modo que la VIP dignifique su forma de vivir y ellos también puedan contribuir con el desarrollo de la ciudad. Este marco de referencia generó elementos contextuales destinados a elaborar una evaluación sobre el programa VIP con base en una muestra de beneficiarios del proyecto Antonia Santos en la ciudad de Tunja, beneficiaros que no han sido ajenos al desplazamiento, las inclemencias de la naturaleza, el conflicto armado u otras condiciones que los ubican como personas protegidas constitucionalmente.

\section{METODOLOGÍA}

En esta sección se presenta el enfoque investigativo, el grupo objeto de estudio, los procedimientos implementados, los instrumentos usados para recolectar los datos y la manera como se hizo el análisis de la información alusiva al trabajo de campo. Este trabajo se focalizó un enfoque cuantitativo de investigación, apoyado con elementos descriptivos e interpretativos de datos cualitativos centrados en la exégesis textual; en correspondencia con Hernández y Mendoza (2018), cuando se usa métodos cuantitativos, los datos se recogen a través de características (variables) observables con el propósito de evaluar aspectos puntuales referidos a los individuos del grupo objeto de estudio; asimismo, para comprender un poco más sobre la realidad social, de tal grupo conviene complementar con elementos de corte cualitativo destinados a interpretar las percepciones, de los participantes; en este caso, también se elaboraron procesos de cruce de informaciones recolectadas mediante una encuesta aplicada a una muestra de beneficiarios de la VIP y la observación no participante y directa del investigador sobre el fenómeno de estudio en el contexto del proyecto VIP Antonia Santos en la ciudad de Tunja a fin de proporcionar mayor validez a la evaluación realizada sobre la misma (Hernández y Mendoza, 2018).

El grupo de participantes se conformó por 72 cabezas de familia beneficiarios del mencionado proyecto VIP, las viviendas fueron adjudicadas por el estado colombiano; el grupo fue seleccionado usando muestreo por conveniencia, los participantes manifestaron de manera verbal, estar de acuerdo con el consentimiento informado, además, por voluntad propia contestaron las preguntas que se les formularon a través de una encuesta; la cual estuvo constituida por tres secciones: en la primera, se recogió información referida a los siguientes aspectos: ambiente de seguridad en el barrio, estética del entorno de la VIP, características técnicas de las instalaciones eléctricas e hidráulicas, distribución de los espacios interiores, confortabilidad y aireación, calidad de los materiales, amplitud de los espacios públicos, satisfacción de la VIP adjudicada. En la segunda, se incluyeron aspectos como: la vivienda como satisfactor de necesidades, seguridad ambiental, interacción de las redes sociales en el barrio, acceso de los beneficiarios a centros comerciales y de educación, riesgos a la salud y a la vida de las personas, y disponibilidad de zonas verdes. La tercera permitió recoger datos alusivos a: el ingreso mensual, la procedencia antes de poseer la VIP, tipo de ocupación, personas a cargo y la entidad adjudicadora de la VIP. Esta encuesta fue validada a través del criterio de tres jueces expertos, tales jueces hicieron algunas observaciones, las cuales fueron acogidas para mejorar el instrumento; las recomendaciones recibidas se incorporaron a la encuesta antes de ser aplicada a los participantes del grupo de estudio.

En referencia a los procedimientos implementados o ruta de trabajo, en primera instancia, se elaboró un marco referencial-contextual, el cual posibilitaría evaluar las principales características del problema visualizado y en segunda instancia efectuar una discusión centrada en los hallazgos asociados con la VIP en el barrio Antonia Santos. El procesado de los datos se efectuó por medio de la hoja de cálculo Excel complementado con las salidas del software estadístico R. Se elaboró un análisis de tipo exploratorio, descriptivo e interpretativo a partir de los datos recolectados, éste se centró en generar tablas e interpretar porcentajes relacionados con la información proporcionada por los encuestados y la observación directa pero no participante del investigador principal en campo (Mendoza et al., 2020). La exégesis textual de la información recogida complementó ciertos aspectos de los resultados cuantitativos obtenidos, los cuales se contrastan a la luz del marco teórico. Finalmente, emergieron los aspectos asociados con la perspectiva de los beneficiarios sobre la VIP que actualmente habitan.

\section{RESULTADOS Y DISCUSIÓN}

En seguida se pueden observar los resultados de este trabajo investigativo, centrados en las respuestas proporcionadas por los encuestados y relacionadas con la evaluación de la VIP que se les ha adjudicado. En este estudio, la valoración de las características (variables) físicas, ambientales y funcionales de la VIP adjudicada a los beneficiarios, se hizo mediante la siguiente escala: Bueno (3), Regular (2) y Deficiente (1). 
Se encontró que el $55 \%$ de los beneficiarios considera que el ambiente de seguridad en el barrio donde está ubicada su VIP es regular, un $34 \%$ indica que es deficiente y solamente el $11 \%$ lo califica como bueno (ver Tabla 1). Asimismo, el $61 \%$ menciona que, la estética del entorno donde está ubicada su VIP es regular, el $11 \%$ la considera deficiente y solo el $28 \%$ la valora como buena. Por consiguiente, se interpreta que en los beneficiarios prevalece la perspectiva de que su VIP es regular tanto en el ambiente de seguridad como en el aspecto estético; tales valoraciones pueden resultar un tanto subjetivas y atribuibles al simple hecho de vivir en la periferia de la ciudad, ya que la observación directa permitió constatar un sitio tranquilo durante el día y un entorno agradable en comparación con otros barrios cercanos. Estos aspectos guardan relación con lo expuesto por Greif (2015), en el sentido de que en las zonas periféricas, el contexto de los barrios vecinos, el propio barrio y la interacción con diversos grupos sociales pueden crear insatisfacciones similares a las identificadas en la urbanización Antonia Santos.

Tabla 1: Evaluación de características (variables) físicas, ambientales y funcionales de la VIP objeto de estudio

\begin{tabular}{|c|c|c|c|}
\hline Variables & Bueno & Regular & Deficiente \\
\hline Ambiente de seguridad en el barrio Antonia Santos & $11 \%$ & $55 \%$ & $34 \%$ \\
\hline Estética del entorno donde está ubicada la VIP adjudicada & $28 \%$ & $61 \%$ & $11 \%$ \\
\hline Características técnicas de las instalaciones eléctricas de la VIP & $35 \%$ & $58 \%$ & $7 \%$ \\
\hline Características técnicas de las instalaciones hidráulicas de la VIP & $14 \%$ & $71 \%$ & $15 \%$ \\
\hline $\begin{array}{l}\text { Estética exterior de la VIP, grado de satisfacción de las expectativas del } \\
\text { usuario }\end{array}$ & $32 \%$ & $59 \%$ & $9 \%$ \\
\hline Distribución de los espacios en el interior de la VIP adjudicada & $68 \%$ & $27 \%$ & $5 \%$ \\
\hline Percepción del beneficiario sobre la confortabilidad y aireación de la VIP & $34 \%$ & $64 \%$ & $6 \%$ \\
\hline Valoración de la calidad de los materiales de construcción de la VIP & $15 \%$ & $72 \%$ & $13 \%$ \\
\hline Amplitud de las calles (vías) de acceso a la urbanización Antonia Santos & $52 \%$ & $36 \%$ & $12 \%$ \\
\hline Satisfacción del usuario con la VIP que le han adjudicado & $58 \%$ & $36 \%$ & $6 \%$ \\
\hline
\end{tabular}

Por otro lado, un $58 \%$ califica como regulares a las características técnicas de las instalaciones eléctricas, el $7 \%$ las considera deficientes y solamente un $35 \%$ percibe que tales instalaciones son buenas. Adicionalmente, el $71 \%$ considera que las características técnicas de las instalaciones hidráulicas son regulares, el $15 \%$ dice que son deficientes y un 14\% percibe que son buenas; por consiguiente, hay una tendencia a que la VIP sea valorada como regular tanto en las instalaciones eléctricas como hidráulicas; estos hallazgos contrastan con lo mencionado en la Convocatoria No. 038 de 2012 en la cual se garantizó una auditoria permanente de la obras de construcción y en particular de las instalaciones mencionadas; sin embargo, según Ramírez (2020) pueden darse algunas imperfecciones en ciertas viviendas ocasionadas porque los constructores llevan al límite la capacidad de carga de los materiales utilizados.

En referencia a la estética exterior de la VIP, al 59\% le ha llenado sus expectativas de forma regular, al $32 \%$ de forma buena y al $9 \%$ le ha parecido deficiente. Por otra parte, el $68 \%$ de los beneficiarios percibe que la distribución de los espacios al interior de la VIP es buena, el $27 \%$ indica que es regular y el $5 \%$ la considera deficiente; sin embargo, el $64 \%$ percibe que la confortabilidad y la aireación de la VIP es regular, el $30 \%$ menciona que es buena y el $6 \%$ señala que es deficiente. Además, el $72 \%$ considera que los materiales usados en la construcción de su VIP fueron de calidad regular, el 15\% indica que fueron buenos y el $13 \%$ los considera de mala calidad. Se interpreta que en un considerable porcentaje los encuestados tienen la razón. Esta perspectiva también se visibiliza en otros lugares de Colombia ya que según Ramírez (2020) los constructores privados piensan en su lucro al trabajar con el mínimo costo posible; por ejemplo, condiciones similares fueron establecidas por Castaño et al. (2018) en un proyecto con cierta semejanza, desarrollado en Turbo-Antioquia destinado a personas desplazadas residentes en viviendas de interés social.

Sobre la amplitud de las calles de acceso a la urbanización Antonia Santos, el 52\% las valoró como buenas, el $36 \%$ como regulares y el $12 \%$ como deficientes. Estos aspectos están relacionados con la transitabilidad de las personas sobre el vecindario, en este sentido, Grasser et al., (2016) encontraron que tales aspectos podían asociarse positivamente con la infraestructura local y la satisfacción del vecindario, aunque su estudio se haya hecho en otro sitio. Finalmente, el $58 \%$ de los beneficiarios se siente bien satisfecho con su VIP, el $36 \%$ están poco satisfechos y el $6 \%$ están insatisfechos. Así se interpreta que, los beneficiarios han valorado de manera regular los aspectos físicos y ambientales de la VIP; no obstante, consideran que la distribución de los espacios interiores es buena aunque los materiales e instalaciones tanto eléctricas como hidráulicas son regulares. Se interpreta que estos hallazgos tienen relación con los costos de la VIP y la planificación implementada en este tipo de proyectos, en los cuales interesa cumplir con los términos de la convocatoria No. 038 más que en las necesidades particulares de los potenciales beneficiarios. Así entonces, los encuestados manejan una perspectiva de que la VIP adjudicada tiende a ser regular en sus características físicas, ambientales y funcionales, dejándolos parcialmente satisfechos; esto es frecuente en el mercado de 
la vivienda puesto que según Fuentes et al. (2020) la vivienda es un satisfactor de necesidades sujeto diversas dinámicas que impactan el bienestar de los usuarios de diferentes maneras por la elasticidad en los precios y el comportamiento de la economía; además Serrano et al. (2019) agregan que las estrategias empresariales deberían favorecer un poco más a la población vulnerable.

En referencia a la percepción cotidiana de los beneficiarios sobre los servicios característicos de una VIP sustentable, el $60 \%$ considera que su VIP como satisfactor de salud es regular, el $36 \%$ percibe que es buena y el $4 \%$ que es deficiente (ver Tabla 2). El $47 \%$ considera que la seguridad ambiental es regular, el $45 \%$ indica que es deficiente y solamente el $8 \%$ la percibe como buena. Con base en la Tabla 2, el $55 \%$ menciona que la interacción de las redes sociales en el barrio es regular, el $26 \%$ señala que es ineficiente y el $19 \%$ la valora como buena. Un $54 \%$ de los beneficiarios de la VIP opina que el acceso a los centros comerciales es deficiente, el $35 \%$ lo considera regular y el $11 \%$ lo califica como bueno; el cambio, el $52 \%$ consideran que el acceso hacia los centros educativos es bueno, el $41 \%$ opina que es regular y el $7 \%$ es deficiente. Adicionalmente, el $72 \%$ menciona que hay poca (regular) disponibilidad de zonas verdes, el $20 \%$ indica que es insuficiente y un $8 \%$ percibe que hay buena disponibilidad. Finalmente, un $73 \%$ indica que existe un nivel regular de riesgo tanto para la salud como para la vida de las personas que habitan en las VIP del barrio Antonia Santos, un $14 \%$ considera que hay un buen nivel de riesgo que contrasta con otro $13 \%$ de los beneficiarios quienes indican que dicho riesgo es bajo. Así entonces, se interpreta que, en los beneficiarios prevalece una tendencia a valorar como regular a los servicios característicos de una VIP sustentable, estos aspectos contrastan con lo expuesto por Wachsmuth y Angelo (2018) quienes manejan la idea de incluir elementos de sustentabilidad al interior de las urbanizaciones aunque en distintas proporciones.

Tabla 2: Percepción cotidiana de los servicios característicos de una VIP sustentable

\begin{tabular}{|l|c|c|c|}
\hline Variables & Bueno & Regular & Deficiente \\
\hline La vivienda como satisfactor de salud & $36 \%$ & $60 \%$ & $4 \%$ \\
\hline Seguridad ambiental & $8 \%$ & $43 \%$ & $45 \%$ \\
\hline Interacción con redes sociales en el barrio & $19 \%$ & $55 \%$ & $26 \%$ \\
\hline Acceso a centros comerciales & $11 \%$ & $35 \%$ & $54 \%$ \\
\hline Acceso a centros educativos & $52 \%$ & $41 \%$ & $7 \%$ \\
\hline Disponibilidad de zonas verdes & $8 \%$ & $72 \%$ & $20 \%$ \\
\hline Riesgo a la salud y la vida de las personas & $14 \%$ & $73 \%$ & $13 \%$ \\
\hline
\end{tabular}

Otras variables que caracterizaron a los beneficiarios de la VIP fueron: Ingresos mensuales percibidos para el sostenimiento familiar, lugar de procedencia, antes de adquirir la vivienda, número de personas a cargo del beneficiario, y tipo de ocupación. A continuación se describen los resultados referidos a cada una de ellas. Los hallazgos evidenciaron que, el $47 \%$ de los beneficiarios de la VIP perciben un ingreso mensual inferior a un salario mínimo colombiano, el $46 \%$ generan un ingreso igual a un salario mínimo y solamente el $7 \%$ gana más de un salario mínimo. Lo anterior refleja que un alto porcentaje de los beneficiarios posee bajos ingresos, requeridos para satisfacer sus necesidades básicas, lo que limita su resiliencia para mejorar su VIP, situación un tanto diferente a la encontrada por Naef (2020) en dos barrios de Medellín donde los beneficiarios de vivienda presentaron una mayor resiliencia. Respecto al lugar de procedencia, antes de adquirir la VIP, un $46 \%$ de los beneficiarios indicó que, procedía del campo y migró a la ciudad en búsqueda de mejores oportunidades, el $31 \%$ era desplazado como producto del conflicto colombiano, el $26 \%$ era oriundo de la ciudad de Tunja y ninguno era afrodescendiente. Estos hallazgos permiten interpretar que, en un alto porcentaje los beneficiarios han sido desplazados o migrantes desde el sector rural; estos hallazgos moderadamente concuerdan con Camargo et al. (2020), quienes han identificado condiciones semejantes en algunos otros sitios del país en torno a las dinámicas de migración, desplazamiento y soluciones habitacionales urbanas.

Además, el $92 \%$ de los beneficiarios de la VIP mencionaron que, su vivienda fue adjudicada por la nación, el $7 \%$ indicó que el municipio de Tunja y el 1\% señaló que el departamento de Boyacá. Por otro lado, en lo referente al número de personas a cargo del beneficiario de la VIP, se determinó que, el $45 \%$ está a cargo de más de cuatro personas, el $38 \%$ atiende a cuatro individuos, el $16 \%$ a tres personas y el $1 \%$ se responsabiliza por menos de tres individuos. Estos hallazgos, indican que las VIP del barrio Antonia Santos albergan a un número grande de personas en espacios relativamente reducidos, este aspecto refuerza las ideas expresadas por Zhang y Li (2018) en tanto que la vivienda ha de ser amplia y sustentable, sin importar el sitio donde se construya. En lo referente al tipo de ocupación de los beneficiarios de la VIP, se encontró que, el $41 \%$ se desempeñaban como trabajadores independientes, el 32\% eran jornaleros, $18 \%$ correspondían a amas de casa y el $9 \%$ eran empleados. Estos hallazgos reflejan una prevalencia de beneficiarios con tipos de ocupación que generarían ingresos económicos bajos e inestables, quienes para mejorar sus condiciones de vida, han de recurrir a su espíritu resiliente, aunque según Castro et al. (2019) no es fácil desligarse de su condición de ser víctima del conflicto armado o de vivir en un barrio periférico con especial protección constitucional. 
Para acrecentar la discusión, en este trabajo se ha valorado la VIP adjudicada a un grupo de beneficiarios en Tunja; quienes tienen clara la perspectiva de que el Estado colombiano y el municipio de Tunja los protege en alguna medida al proporcionarles una VIP, la cual según Mejía (2012) se espera que dignifique y mejore su actual condición de vida; no obstante, un alto porcentaje de beneficiarios considera que su vivienda actual fue adjudicada con características físicas, ambientales y funcionales en un estado regular, aún requiere ser mejorada en cuanto a sus características para que se constituya en vivienda sustentable y les permita disfrutar de ambientes más dignos para el núcleo familiar, en el cual acudiendo a su propia resiliencia están superando el estado de migración, desplazamiento forzado o pobreza que por años los han aquejado. En este contexto, el Estado colombiano ha de seguirlos apoyando económicamente, en tanto que cerca del $91 \%$ son jornaleros, cabezas de hogar o trabajadores independientes con un ingreso menor o igual a un salario mínimo, con el cual deben sufragar los gastos para cuatro o más personas que habitan cada VIP adjudicada.

Los aspectos característicos de una VIP construida con 70 salarios mínimos, han generado impactos de diversa índole, entre ellos, en las dimensiones económica, física, ambiental y social de los beneficiarios; en un gran número de casos se tiene una perspectiva positiva sobre su VIP pero en otros pocos tal perspectiva es negativa, como consecuencia de estados regulares o deficientes en su infra-estructura física o en el entorno medioambiental e interacción con los miembros del vecindario; de acuerdo con Adewale et al. (2017) esta perspectiva también es observable en otros lugares del continente africano aunque con diferente enfoque cultural; en este contexto, los representantes del Estado colombiano y de las empresas constructoras de la VIP deben cumplir de mejor manera su función social de proveer de una VIP con mayores controles de calidad para que los usuarios puedan mejorar su condición actual de vida, por ejemplo, algunas VIP requirieron arreglos en las conexiones eléctricas e hidráulicas, mesón de la cocina y baños, en otras, corregir problemas de estética (pintura) tanto en el ambiente interno como externo para que las VIP no sean percibidas por los beneficiarios y el ciudadano común como barrios marginales, o como lo indican Vilar y Cortés (2016) sean consideradas como tugurios en la periferia para el caso de la ciudad de Medellín. Siguiendo a Castro et al. (2019), si estos aspectos no se mejoran puedan ocasionar impactos psicosociales en las personas que habitan la VIP así haya sido entregada de forma gratuita.

Por otra parte, los hallazgos evidenciaron la existencia de una asociación moderada e inversa entre ingresos que percibe cada hogar y el número de integrantes de cada familia beneficiaria de la VIP (prueba chi_cuadrado con P-valor = 0.427); por consiguiente, los hogares con mayor número de personas a su cargo gastará menos en mejoramiento de sus VIP, lo cual significa que, en un alto porcentaje los beneficiarios seguirán habitando sus viviendas en similares condiciones a las del momento en que les fueron entregadas o en condiciones de deterioro a casusa de su uso con el transcurrir del tiempo; estos resultados concuerdan con lo expresado por García et al., (2018) aunque en otras proporciones, en el sentido de que cualquier gasto relacionado con la mejora en la VIP irá en detrimento de la economía familiar y una marcada mengua en la satisfacción de las necesidades básicas de la familia. Por otra parte, la ubicación del barrio no fue la mejor y en su infraestructura se perciben dificultades para un acceso pronto a los centros comerciales o a los lugares de trabajo de los beneficiarios, aspecto que ha creado cierta insatisfacción familiar al habitar este barrio de Tunja y que guarda relación con lo expuesto por Mendoza et al., (2020) en aspectos similares detectados en otros proyectos de vivienda de interés social en este municipio colombiano; además, la perspectiva de los encuestados en Antonia Santos concuerda con lo expresado por Libertun (2018), en cuanto a que la vivienda ubicada en zona periferia puede generar segregación e insatisfacción residencial, debilitar los vínculos sociales con el resto de la ciudad e incrementar los tiempos para que las personas se trasladen desde su residencia a los lugares trabajo.

\section{CONCLUSIONES}

Con el trabajo efectuado y los hallazgos obtenidos, se plantearon las siguientes conclusiones asociadas con la evaluación realizada por los beneficiarios de la VIP: 1) La vivienda que les fue adjudicada en el barrio Antonia Santos fue mayoritariamente valorada como regular, en las variables tanto físicas como ambientales; sin embargo, tienen una perspectiva positiva que la distribución de los espacios interiores es buena aunque los materiales de construcción y las instalaciones tanto eléctricas como hidráulicas pueden valorarse como regulares. 2) Los servicios característicos de una VIP sustentable también son regulares; no obstante consideran que la urbanización posee un acceso bueno hacia los centros educativos pero deficitarios para el acceso a sus sitios cotidianos de trabajo y los centros comerciales que frecuentan. 3) El estado actual de las VIP no es el mejor, varias viviendas se han deteriorado con el uso y algunas permanecen en similares condiciones a las que fueron entregadas a los beneficiarios, estado que tampoco se presta para mantener un ambiente estético tanto en el ambiente exterior como interior de la urbanización. 4) Los ingresos de las familias beneficiarias no permiten el mejoramiento de la VIP porque resulta perentorio solventar primero sus necesidades básicas, en particular para las familias con cuatro o más integrantes. Por consiguiente, la VIP ha de mejorarse en distintos aspectos antes de que sea adjudicada a los potenciales beneficiarios. 


\section{REFERENCIAS}

Adewale, B.A., Ibem, E.O., Amole, S. A., y Adeboye, A.B., Place attachment in Nigerian urban slums: Evidence from inner-city Ibadan, https://doi.org/10.1016/j.cities.2020.102902, Cities, 107, 102902 (2020)

Brito, C., Pitre, R., y Cardona, D., Clima organizacional y su influencia en el Desempeño del personal en una Empresa de Servicio, http://dx.doi.org/10.4067/S0718-07642020000100141, Información Tecnológica, 31(1), 141-148 (2020)

Caldeira, T.P., Peripheral urbanization: Autoconstruction, Transversal logics, and Politics in cities of the Global south, Environment and Planning D: Society and Space, 35(1), 3-20 (2017)

Camargo, G., Sampayo, A.M., y otros 4 autores, A., Exploring the dynamics of Migration, Armed conflict, Urbanization, and Anthropogenic change in Colombia, https://doi.org/10.1371/journal.pone.0242266, Plos one, 15(11), 1-18, (2020)

Cardona, S., Escobar, D., y Moncada, C., Análisis de Ordenamiento de la Movilidad en el Bulevar gastronómico de Milán, Manizales (Colombia), http://dx.doi.org/10.4067/S0718-07642020000100301, Información Tecnológica, 31(1) $301-310$ (2020)

Castaño, Y., Atehortua, S., y López, Y., Condiciones socio-habitacionales y Morbilidad percibida de Desplazados internos residentes en Viviendas de Interés Social en Turbo, Antioquia, Revista Ciencias de la Salud, 16(2), 237-261 (2018)

Castles, S., Las Fuerzas tras la Migración global, Revista Mexicana de Ciencias Políticas y Sociales, 59(220), 235-259 (2014)

Castro, L., Rattner, M., y otros 4 autores, A contextual adaptation of the Unified Protocol for the Transdiagnostic

Treatment of Emotional Disorders in victims of the armed conflict in Colombia, Cognitive and Behavioral Practice, 26(2), 351-365 (2019)

Ceballos, O., y Caquimbo, S., Las 100.000 Viviendas gratuitas: Contradicciones no superadas en la Política pública de vivienda en Colombia, Cuadernos de vivienda y urbanismo, 7 (13), 6-8 (2014)

Chávez, M., Pérez, D.D., y Serrano, M.F., Impact on the direct costs of Social Interest and Priority interest Housing by including new Construction Standards: Cali case, Dyna, 85 (206), 31-38 (2018)

De La Ossa, E.M.D., Pérez, D.F., y Prado, A.L.R., Competencias parentales y Resiliencia infantil en contexto de desplazamiento en Colombia, Revista Prisma Social, 20, 227-253 (2018)

Fuentes, H.J., Contreras, L.E., y Rodríguez, J.I., Un análisis del Mercado de Vivienda en Colombia 1998-2020, Revista Espacios, 41(31), 267-278 (2020)

García, S., Smith, H., y Calderón, E., Contemporary tendencies in Colombian urban planning: The case of the 'Planes Parciales' in Medellín, International Planning Studies, 23(4), 355-375 (2018)

Grasser, G., Titze, S., y Stronegger, W., Are residents of high-walkable Areas satisfied with their Neighbourhood?, Journal of Public Health, 24, 469-476 (2016)

Greif, M., The intersection of homeownership, Race and Neighbourhood context: Implications for Neighbourhood satisfaction, Urban Studies (Sage Publications, Ltd.), 52(1), 50-70 (2015)

Hernández, S.R., y Mendoza, C., Metodología de la Investigación. Las rutas Cuantitativa, Cualitativa y Mixta, Editorial Mc Graw Hill Education, Ciudad de México, México (2018)

Hidalgo, A., El Papel de la Vivienda en la Configuración Urbana de las Periferias: caso de Tunja-Colombia 1907-2007. Cuadernos de vivienda y urbanismo, 1(1), 12-43 (2008)

Libertun, N., The social Housing burden: comparing Households at the Periphery and the Centre of cities in Brazil, Colombia, and Mexico, https://doi.org/10.1080/19491247.2 017.1298366, International Journal of Housing Policy, 18(2), 177-203 (2018)

Malerba, D., Poverty alleviation and local Environmental degradation: An empirical analysis in Colombia, https://doi.org/10.1016/j.worlddev.2019.104776, World Development, 127, 104776 (2020)

Mejía, E.M., Habitabilidad de la Vivienda social en Edificios para Población reasentada: El caso de Medellín, Colombia, EURE (Santiago), 38(114), 203-227 (2012)

Mendoza, J.M., Burbano, V.M., y Mendoza, H.H., Enseñanzas atribuibles a los Procesos de Adjudicación de Vivienda de Interés Social en una ciudad colombiana: grado de satisfacción del usuario, Información Tecnológica, 31(5), 119-118 (2020)

Murray, C., y Clapham, D., Housing policies in Latin America: Overview of the four largest Economies, International Journal of Housing Policy, 15(3), 347-364 (2015)

Naef, P., Resilience as a City Brand: The Cases of the Comuna 13 and Moravia in Medellin, Colombia, https://doi.org/10.3390/su12208469, Sustainability, 12(20), 1-21, (2020)

Ramírez, W. G., Calidad de la Vivienda en Colombia. El Caso de ciudades dentro de la Ciudad. Cuadernos de Vivienda y Urbanismo, 13, 1-22 (2020)

Rodríguez, C. P., La Accesibilidad en debate:¿ localizar Vivienda Social en áreas centrales de Bogotá?. Cuadernos de Vivienda y Urbanismo, 13, 1-18 (2020) 
Serrano, A.M., Tiuzo, S.C., y Martínez, M.S., Estrategias Empresariales para una Población Vulnerable en Colombia, Información tecnológica, 30(6), 147-156 (2019)

Vilar, K., y Cortes, I., Urban design and Social Capital Inslums. Case study: Moravia's neighborhood, Medellin, 20042014, Procedia-social and behavioral sciences, 216, 56-67 (2016)

Von-Breymann, H., Indicadores de percepción de la Calidad del entorno Residencial: una Herramienta para la Planificación Urbana, Revista INVI, 34(95), 101-132 (2019)

Wachsmuth, D., y Angelo, H., Green and gray: New ideologies of Nature in Urban sustainability Policy, Annals of the American Association of Geographers, 108(4), 1038-1056 (2018)

Zhang, X., y Li, H., Urban Resilience and urban Sustainability: What we know and what do not know?, Cities, 72, 141-148 (2018) 
